\title{
AVALIAÇÃO DA SELETIVIDADE E EFICÁCIA DE MISTURAS FORMULADAS DE BENTAZON + ACIFLUORFEN EM SOJA
}

\author{
Laca-Buendía, J. P. ${ }^{(1)}$; Lara, J. F. R. ${ }^{(2)}$ \\ ${ }^{1}$ Eng. Agr., M. Sc., Pesq./EPAMIG/CTTP/FEGV Caixa Postal, 351 Uberaba CEP 38060-040 Belo \\ Horizonte, MG. E-mail: juliolaca@epamiguberaba.com.br \\ ${ }^{2}$ Biólogo/ Pesq./EPAMIG/CTCO/FESR Caixa Postal, 295 CEP 35701-970 Sete Lagoas, MG
}

\section{RESUMO}

O objetivo do ensaio foi avaliar a seletividade e eficácia de misturas formuladas dos herbicidas bentazon $400 \mathrm{~g} / \mathrm{L}+$ acifluorfen sal de sódio $170 \mathrm{~g} / \mathrm{L}$; bentazon 400 $\mathrm{g} / \mathrm{L}+$ acifluorfen sal de sódio $140 \mathrm{~g} / \mathrm{L}$ e bentazon $300 \mathrm{~g} / \mathrm{L}+$ acifluorfen sal de sódio 80 $\mathrm{g} / \mathrm{L}$, em pós-emergência na cultura da soja (cv. Doko). O solo foi um LatossoloVermelho-Escuro, textura argilo-siltoso, no município de Prudente de Morais, MG. Os tratamentos testados foram: $[480+204]$ e $[600+255] \mathrm{g} / \mathrm{ha}$ do i. a., com e sem mistura de óleo mineral; $[480+168]$ e $[600+210] \mathrm{g} / \mathrm{ha}$ do i. a., com e sem mistura de óleo mineral; $[600+160 \mathrm{~g} / \mathrm{la}$ do i. a.; testemunha capinada e testemunha sem capina. Todos os herbicidas testados independente da dose a adição de óleo mineral 0,25\% v/v apresentaram controle eficiente de Amaranthus hybridus (caruru), Galinsoga parviflora (mentrasto) e Solanum americanum (maria-pretinha). Não foram observadas diferenças significativas entre produtividade nos diferentes tratamentos químicos testados com a testemunha capinada, sendo que a interferência das plantas daninhas diminuiu o rendimento em $1445 \mathrm{~kg} / \mathrm{ha}$ em média. As aplicações dos herbicidas não afetou a população final, altura da planta, altura de inserção da primeira vagem e o peso de 100 sementes. Todas as misturas formuladas de herbicidas testadas foram seletivas para a cultura da soja.

Palavras Chave: controle químico, Amaranthus hybridus, Galinsoga parviflora, Solanum americanum, Glycine max.

\section{EVALUATION OF SELECTIVITY AND EFFICACY OF FORMULATION MIXTURES OF BENTAZON AND ACIFLUORFEN APPLIED TO SOYBEANS}

\begin{abstract}
A trial was carried out to test the efficacy and selectivity of different formulations and doses of bentazon with acifluorfen, applied in post emergence of soybean (cv. Doko). The following treatments were tested in a sandy Latosoil, in Prudente de Morais, Brazil: bentazon+acifluorfen $(480+204 \mathrm{~g} / \mathrm{ha}$ and $600+255 \mathrm{~g} / \mathrm{ha}$ ) with or without mineral oil $(0,25 \% \mathrm{v} / \mathrm{v})$; bentazon+acifluorfen $(480+168 \mathrm{~g} / \mathrm{ha}$ and $600+210 \mathrm{~g} / \mathrm{ha})$ with or without mineral oil $(0,25 \% \mathrm{v} / \mathrm{v})$; and bentazon +acifluorfen $600+160 \mathrm{~g} / \mathrm{ha}$ and weeded and no weeding checks. Independently of the doses and formulations (with or without mineral oil) all chemicals provided efficient control of Amaranthus hybridus, Galinsoga parviflora and Solanum americanum. Significant differences were not observed among the productivities for the several chemical treatments, but reduced the interference of weeds caused an average decrease in yield of $1445 \mathrm{~kg} / \mathrm{ha}$ in relation to the weed check. These was no difference between treatments
\end{abstract}


concerning plant density, plant height, height of first pod and weight of 100 seeds. All tested treatments were selective for the soybean

Key words: control chemical, Amaranthus hybridus, Galinsoga parviflora, Solanum americanum, Glycime max.

\section{INTRODUÇÃO}

O Brasil foi o segundo exportador mundial de soja, com as receitas cambiais de US\$ 3,796 bilhões em 1995 (Cargill, 1997).

A produção brasileira de soja em 1995/96 superou 23 milhões de toneladas colhidas numa área de 10,710 milhões de hectares e com produtividade de $2.154 \mathrm{~kg} / \mathrm{ha}$ (Cargill, 1997).

A concorrência das plantas daninhas com a cultura, reduz a produção em cerca de $90 \%$ ( Blanco, et al., 1973).

Para o controle químico das plantas daninhas na cultura de soja, com o surgimento de herbicidas de aplicação na pós-emergência e altamente seletivos, vem sendo desenvolvidos misturas de herbicidas com eficiente controle.

Vários pesquisadores tem estudado herbicidas aplicados em pós-emergência para o controle das plantas daninhas, conforme pode ser observado pelos trabalhos desenvolvidos por: Netto et al. (1991); Stroher \& Haden (1993); Pereira \& Bazoni (1995); Cheata et al. (1997); Duarte et al. (1997); Laca-Buendía \& Lara (1997); LacaBuendía \& Nislara (1997); Osipe et al. (1997); Zagonel (1997) e Zagonel \& Venancio (1997).

O objetivo do presente ensaio foi estudar a eficácia de Volt [bentazon $400 \mathrm{~g} / \mathrm{L}$ do i. a. +acifluorfen sal de sódio $170 \mathrm{~g} / \mathrm{L}$ do i. a.] e Gunner [bentazon $400 \mathrm{~g} / \mathrm{L}$ do i. a.+acifluorfen sal de sódio $140 \mathrm{~g} / \mathrm{L}$ do i. a.], com e sem mistura de óleo mineral $(0,25 \% \mathrm{v} / \mathrm{v})$, aplicados em pós-emergência, no controle das plantas daninhas e verificar sua seletividade sobre a cultura da soja, em área de Cerrado.

\section{MATERIAL E MÉTODOS}

O presente ensaio de campo foi instalado, no município de Prudente de Morais, MG, no ano agrícola de 1996/97 num solo Latossolo-Vermelho-Escuro, textura argilosiltoso, contendo $47 \%$ de argila, $45 \%$ de silte e $8 \%$ de areia, $\mathrm{pH}$ (água) $=7,3 ; 17$ e $4 \%$ de matéria orgânica.

A cultivar usada foi Doko, plantada em 08/11/1996, utilizando-se o espaçamento de $0,5 \mathrm{~m}$. entre fileiras, com semeadura manual e densidade de 25 sementes por metro linear. A adubação de plantio usada foi de.400 kg /ha da fórmula 0:20:20 de NPK.

$\mathrm{O}$ ensaio foi instalado no desenho experimental de blocos casualizados, com quatro repetições em parcelas de quatro fileiras de $7,0 \mathrm{~m}$ de comprimento, dando uma área total de $14,0 \mathrm{~m}^{2}$, tendo uma área útil de avaliação de $1,0 \mathrm{~m}$ x $6,0 \mathrm{~m}=6,0 \mathrm{~m}^{2}$.

As aplicações dos herbicidas (Tabela 1) foram realizadas em 30/11/96, em pósemergência da cultura da soja no estágio de três a quatro trifólios e quando as plantas daninhas latifoliadas apresentavam duas a quatro folhas, utilizando pulverizador costal pressurizado a $\mathrm{CO}_{2}$, dotado de barra, com quatro pontas do tipo leque $110.04 \mathrm{VS}$ a 0,5 $\mathrm{m}$ de altura do solo, com vazão de $250 \mathrm{~L} / \mathrm{ha}$, usando-se uma pressão constante de 2,8 $\mathrm{kg} / \mathrm{cm}^{3}$. As condições climáticas (Tabela 2) no momento da aplicação foram caracterizadas pela temperatura do ar de $20,3{ }^{\circ} \mathrm{c}$ e umidade relativa do ar de $74 \%$. As aplicações foram realizadas entre $16 \mathrm{~h} 00 \mathrm{~min}$ e $18 \mathrm{~h} 00 \mathrm{~min}$, sendo que o céu 
apresentava-se parcialmente nublado e o solo úmido e sem vento por ocasião da aplicação.

Foram realizadas duas capinas manuais nas testemunhas capinadas, em 12/01/97 e $22 / 02 / 97$.

Tabela 1- Tratamentos utilizados no experimento.

\begin{tabular}{|c|c|c|c|c|}
\hline \multirow[t]{2}{*}{ Produto Técnico } & \multirow{2}{*}{$\begin{array}{l}\text { Nome } \\
\text { comercial }\end{array}$} & \multicolumn{2}{|c|}{ Doses do produto } & \multirow{2}{*}{$\begin{array}{c}\text { Concentração } \\
\text { g/L }\end{array}$} \\
\hline & & $\begin{array}{l}\text { Produto } \\
\text { técnico } \\
\text { g/ha i. a. }\end{array}$ & $\begin{array}{l}\text { Produto } \\
\text { comercial } \\
\text { L/ha p. c. }\end{array}$ & \\
\hline bentazon+acifluorfen sal de sódio & Volt & {$[480+204]$} & 1,2 & 570 \\
\hline $\begin{array}{l}\text { bentazon+acifluorfen } \\
\text { sódio+óleo mineral }^{(*)}\end{array} \quad$ sal $\quad$ de & Volt+Assist & {$[480+204]$} & $1,2+0,25 \% \mathrm{~V} / \mathrm{V}$ & $570+189$ \\
\hline bentazon+acifluorfen sal de sódio & Volt & {$[600+255]$} & 1,5 & 570 \\
\hline $\begin{array}{l}\text { bentazon } 400+\text { acifluorfen } 170+ \\
\text { oleo mineral }^{(*)}\end{array}$ & Volt+Assist & {$[600+255]$} & $1,5+0,25 \% \mathrm{~V} / \mathrm{V}$ & $570+189$ \\
\hline bentazon 400+acifluorfen 140 & Gunner & {$[480+168]$} & 1,2 & 540 \\
\hline $\begin{array}{l}\text { bentazon } 400+\text { acifluorfen } 140+ \\
\text { óleo mineral }{ }^{(*)}\end{array}$ & Gunner+Assist & {$[480+168]$} & $1,2+0,25 \% \mathrm{~V} / \mathrm{V}$ & $540+189$ \\
\hline bentazon 400+acifluorfen 140 & Gunner & {$[600+140]$} & 1,5 & 540 \\
\hline $\begin{array}{l}\text { bentazon } 400+\text { acifluorfen } 140+ \\
\text { oleo mineral }^{(*)}\end{array}$ & Gunner+Assist & {$[600+140]$} & $1,5+0,25 \% \mathrm{~V} / \mathrm{V}$ & $540+189$ \\
\hline Bentazon+acifluorfen sal de sódio & Doble & {$[600+180]$} & 2,0 & 380 \\
\hline Testemunha capinada & --- & --- & -- & --- \\
\hline Testemunha sem capina & --- & --- & -- & --- \\
\hline
\end{tabular}

Tabela 2- Precipitações pluviais, em mm, durante a condução do ensaio.

\begin{tabular}{l|c|c|c|c}
\hline \multicolumn{1}{c|}{ Mês } & $1^{\underline{0}}$ Decêndio & $2^{\underline{0}}$ Decêndio & $3^{0}$ Decêndio & Total \\
\hline Novembro & 177,26 & 99,12 & 208,64 & 485,02 \\
Dezembro & 69,48 & 98,48 & 144,22 & 312,35 \\
Janeiro & 349,13 & 39,44 & 12,42 & 400,99 \\
Fevereiro & 23,82 & 0,00 & 130,08 & 153,90 \\
Março & 114,24 & 33,68 & 0,00 & 147,92 \\
\hline Total & & & & $1.500,18$ \\
\hline
\end{tabular}

O grau de fitotoxicidade foi avaliado através de escala visual EWRC (1 a 9 ), aos 7 e 15 dias após a aplicação (DAA), onde $1=$ nenhuma injúria e $9=$ morte da planta.

A avaliação para controle das plantas daninhas empregou-se a escala visual de percentagem de controle $(0 \%$ a $100 \%)$, onde $0 \%=$ nenhum controle (nulo) e $100 \%=$ controle total (excelente). As avaliações foram efetuadas aos 15, 21 e 35 e nas parcelas sem capina foram realizadas as contagens de todos os indivíduos presentes por espécies numa área de $1,0 \mathrm{~m}^{2}$.

Antes da colheita foi realizada uma avaliação visual para colheita mecânica segundo a escala: 1- impossível (100\% a $80 \%$ ) de infestação), 2- alta infestação (79\% a $60 \%$ de infestação), 3- infestação razoável (59\% a $40 \%$ de infestação), 4- boa colheita (39\% a 20\% de infestação) e 5- excelente (òtima) (19\% a 0\% de infestação).

Quando as plantas de soja atingiram a maturação de grãos foi realizada a colheita (02/04/97), onde se realizou a pesagem dos grãos da área útil e contou-se o número de indivíduos $/ 7,0 \mathrm{~m}^{2}$ de cada parcela. A altura média das plantas e a altura de inserção da primeira vagem foram tomadas de 10 plantas, ao acaso, dentro das duas 
fileiras centrais de cada parcela. Foram escolhidas, ao acaso, 100 sementes para determinar seu peso em cada parcela.

Os dados obtidos foram submetidos a análise de variância pelo teste de $\mathrm{F}$ e as médias comparadas pelo teste de Tukey a nível de 5\% de probabilidade.

\section{RESULTADOS E DISCUSSÃO}

As espécies encontradas foram: Amaranthus hybridus (caruru), com 14 indivíduos $/ \mathrm{m}^{2}$, Galinsoga parviflora (mentrasto), com 12 indivíduos $/ \mathrm{m}^{2}$ e Solanum americanum (maria-pretinha), com 11,25 indivíduos $/ \mathrm{m}^{2}$.

De acordo com os resultados, pode-se verificar que pela análise da variância realizada para a população de plantas na colheita e na altura de inserção da primeira vagem, não foram encontradas diferenças significativas entre as misturas formuladas dos herbicidas avaliadas e nas testemunhas (Tabela 3 ).

Na produção de grãos, altura de planta, número de vagens por parcela e peso de 100 sementes, houve diferenças significativas, verificando-se entretanto, que nenhum das misturas formuladas dos herbicidas testadas apresentou reduções significativas em relação à testemunha capinada. A interferência de plantas daninhas diminuiu o rendimento em $1445 \mathrm{~kg} / \mathrm{ha}$ (Tabela 3 ).

Nenhuma das misturas formuladas dos herbicidas nas doses testadas apresentou efeitos de fitotoxicidade nas plantas de soja (Tabela 3).

Para o controle do caruru, mentrasto e maria-pretinha, foi observado que todas as misturas formuladas dos herbicidas, independente das doses testadas ou do acréscimo de óleo mineral apresentaram índices de controle superiores aos $95 \%$ até 35 dias após a aplicação (Tabela 4).

Todas as misturas de herbicidas formuladas isoladas e em mistura com óleo mineral, nas doses testadas, mostraram controle eficaz de Amaranthus hybridus (caruru), Galinsoga parviflora (mentrasto) e Solanum americanum (maria-pretinha). Todas as mistura de herbicidas formuladas isoladas e em mistura com óleo mineral, nas doses testadas são seletivas para a cultura da soja.

\section{BIBLIOGRAFIA CONSULTADA}

BLANCO, H. G.; et al. Observação sobre o período em que as plantas daninhas competem com a soja (Glycine $\max$ (L.) Merrill. O Biológico, v. 39, n. 2, 1973. p. $31-35$.

CHEATA, A. N.; FORNAROLLI, D. A.; PALMA, D. V. Aplicações de dois herbicidas do grupo dos imidazoles na cultura da soja (Glycine max (L.) Merrill). In CONGRESSO BRASILIREIRO DA CIÊNCIA DAS PLANTAS DANINHAS, 21., 1997, Caxambu, MG. Resumos... Viçosa, MG:SBCPD, 1997, p. 62.

DUARTE, N. de F.; SILVA, J. B. da; ARCHANGELO, E. R.; OLIVEIRA, R. de. Avaliação de imazethapyr e imazomox no controle pós-emergente de plantas daninhas na cultura da soja. In: CONGRESSO BRASILIREIRO DA CIENCIA DAS PLANTAS DANINHAS, 21., 1997, Caxambu, MG. Resumos... Viçosa, MG:SBCPD, 1997, p. 77. 
LACA-BUENDÍA, J. P.; LARA, L. F. R. Eficácia e seletividade do oxasulfuron, aplicado na pós-emergência para o controle de plantas daninhas latifoliadas anuais na cultura da soja (Glycine max (L.) Merrill). In: CONGRESSO BRASILIREIRO DA CIÊNCIA DAS PLANTAS DANINHAS, 21., 1997, Caxambu, MG. Resumos... Viçosa, MG:SBCPD, 1997, p. 101.

LACA-BUENDÍA, J. P.; NISRALA, M. A. Atividade biológica e seletividade do oxasulfuron, aplicado na pós-emergência para o controle de plantas daninhas latifoliadas anuais na cultura da soja (Glycine $\max$ (L.) Merrill). In: LACABUENDÍA, J. P.; LARA, L. F. R. Eficácia e seletividade do oxasulfuron, aplicado na pós-emergência para o controle de plantas daninhas latifoliadas anuais na cultura da soja (Glycine max (L.0 Merrill). In: CONGRESSO BRASILIREIRO DA CIÊNCIA DAS PLANTAS DANINHAS, 21., 1997, Caxambu, MG. Resumos... Viçosa, MG:SBCPD, 1997, p. 101.

NETTO, F. S.; ALMEIDA, F. L. S.; PASSINI, T. Eficácia de herbicidas no controle de plantas latifoliadas na cultura da soja.In:CONGRESSO BRASILEIRO DE HERBICIDAS E PLANTAS DANINHAS, 18., 1991,Londrina. Resumos...Londrina: SBHED, 1991, p. 47.

OSIPE, R.; NISHIMURA, M.; LOPES, D. Avaliação da eficiência e seletividade de herbicidas aplicados em misturas de tanque, em pós-emergência na cultura da soja. In: CONGRESSO BRASILIREIRO DA CIÊNCIA DAS PLANTAS DANINHAS, 21., 1997, Caxambu, MG. Resumos... Viçosa, MG:SBCPD, 1997, p. 115.

PEREIRA, F. de A. R.; BAZONI, R. Avaliação de herbicidas na cultura de soja em áreas de Cerrado de Mato Grosso do Sul. Campo Grande, MS. EMPAER, 1995. 38p. (EMPAER-MS. Documentos, 45).

SOJA: Uma caminhada sem fim: Como a soja conquistou o mundo. E o Brasil. [Campinas]: Fundação Cargill, [1997?], 95p.

STROHER, I. H.; HADEN, E. Avaliação da eficiência e seletividade de Bentazon em mistura com outros herbicidas latifolicidas. In: CONGRESSO BRASILEIRO DE HERBICIDAS E PLANTAS DANINHAS, 19., 1993, Londrina. Resumos dos trabalhos técnicos-científicos. Londrina: SBHED, 1993, p. 94-96.

ZAGONEL, J. Controle de plantas daninhas na cultura da soja com oxasulfuron e as misturas oxasulfuron+imazethapyr e oxasulfuron+imazamox. In: CONGRESSO BRASILIREIRO DA CIÊNCIA DAS PLANTAS DANINHAS, 21., 1997, Caxambu, MG. Resumos... Viçosa, MG:SBCPD, 1997, p. 142.

ZAGONEL, J.; KAMAIKOGA, A. T. M. Avaliação do oxasulfuron aplicado isoladamente e em mistura com acifluorfen e bentazon no controle de plantas daninhas na cultura da soja. In: CONGRESSO BRASILIREIRO DA CIENCIA DAS PLANTAS DANINHAS, 21., 1997, Caxambu, MG. Resumos... Viçosa, MG:SBCPD, 1997, p. 101. 
Tabela 3- Resultados obtidos na avaliação da seletividade e eficácia de misturas formuladas de bentazon e acifluorfen em soja. Prudente de Morais, MG 1996/97.

\begin{tabular}{|c|c|c|c|c|c|c|c|c|c|}
\hline \multirow[t]{2}{*}{ Tratamentos } & \multirow{2}{*}{$\begin{array}{l}\text { Doses } \\
\text { i. a. }(\mathrm{g} / \mathrm{ha})\end{array}$} & \multirow{2}{*}{$\begin{array}{c}\text { Stand } \\
\text { Final } \\
\left(7,0 \mathrm{~m}^{2}\right)\end{array}$} & \multirow{2}{*}{$\begin{array}{l}\text { Produção } \\
\text { (kg/ha) }\end{array}$} & \multirow{2}{*}{$\begin{array}{c}\text { Altura da } \\
\text { Planta } \\
(\mathrm{Cm})\end{array}$} & \multirow{2}{*}{$\begin{array}{c}\text { Altura } \\
\text { Inserção } \\
1^{\mathrm{a}} \text { vagem } \\
(\mathrm{cm})\end{array}$} & \multirow{2}{*}{$\begin{array}{c}\text { Peso de } \\
100 \\
\text { sementes } \\
(\mathrm{g})\end{array}$} & \multicolumn{2}{|c|}{ Fitotoxicidade (1 a 9) } & \multirow{2}{*}{$\begin{array}{l}\text { Colheita } \\
\text { mecânica } \\
\text { (1 a 5) }\end{array}$} \\
\hline & & & & & & & $7 \mathrm{DAA}^{(1)}$ & $15 \mathrm{DAA}$ & \\
\hline Volt & {$[480+204]$} & $239,8 \mathrm{a}^{(2)}$ & $2310 a$ & $102,8 \mathrm{a}$ & 29,3 & $19,8 \mathrm{a}$ & 1,0 & 1,0 & $3,6 \mathrm{~b}$ \\
\hline Volt+Assist & {$[480+204]+0,25 \% \mathrm{v} / \mathrm{v}$} & $241,8 \mathrm{a}$ & $2285 a$ & $104,9 \mathrm{a}$ & 26,8 & $19,2 \mathrm{ab}$ & 1,0 & 1,0 & $4,1 \mathrm{ab}$ \\
\hline Volt & {$[600+255]$} & $242,2 a$ & $2290 a$ & $102,3 a$ & 29,0 & $18,9 \mathrm{ab}$ & 1,0 & 1,0 & $3,9 \mathrm{~b}$ \\
\hline Volt+Assist & {$[600+255]+0,25 \% \mathrm{v} / \mathrm{v}$} & $245,0 \mathrm{a}$ & $2245 a$ & $105,6 a$ & 27,4 & $19,4 \mathrm{ab}$ & 1,0 & 1,0 & $4,2 \mathrm{ab}$ \\
\hline Gunner & {$[480+168]$} & $247,0 \mathrm{a}$ & $2278 \mathrm{a}$ & $104,4 a$ & 27,4 & $18,9 \mathrm{ab}$ & 1,0 & 1,0 & $3,9 \mathrm{~b}$ \\
\hline Gunner+Assist & {$[480+168]+0,25 \% \mathrm{v} / \mathrm{v}$} & $245,5 a$ & $2258 \mathrm{a}$ & $104,2 a$ & 30,4 & $19,6 a b$ & 1,0 & 1,0 & $4,0 \mathrm{~b}$ \\
\hline Gunner & {$[600+210]$} & $243,8 \mathrm{a}$ & $2325 a$ & $100,2 \mathrm{a}$ & 29,2 & $19,6 a b$ & 1,0 & 1,0 & $4,0 \mathrm{~b}$ \\
\hline Gunner+Assist & {$[600+210]+0,25 \% \mathrm{v} / \mathrm{v}$} & $242,2 a$ & $2290 \mathrm{a}$ & $103,6 a$ & 28,7 & $18,9 \mathrm{ab}$ & 1,0 & 1,0 & $4,4 \mathrm{ab}$ \\
\hline Doble & {$[600+160]$} & $247,5 \mathrm{a}$ & $2285 a$ & $100,5 a$ & 29,4 & $18,9 \mathrm{ab}$ & 1,0 & 1,0 & $4,2 \mathrm{ab}$ \\
\hline $\begin{array}{l}\text { Testemunha } \\
\text { capinada }\end{array}$ & --- & $244,8 \mathrm{a}$ & $2285 a$ & $102,9 \mathrm{a}$ & 28,7 & $19,2 \mathrm{ab}$ & 1,0 & 1,0 & $5,0 \mathrm{a}$ \\
\hline $\begin{array}{l}\text { Testemunha sem } \\
\text { capina }\end{array}$ & ---- & $42,2 b$ & $840 b$ & $77,9 b$ & 29,1 & $19,4 a b$ & 1,0 & 1,0 & $1,0 \mathrm{c}$ \\
\hline Média Geral & & 225,6 & 2154 & 100,9 & 28,6 & 17,9 & 1,0 & 1,0 & 3,8 \\
\hline Valor de F & & $636,8 * *$ & $862,2 * *$ & $25,6 * *$ & $1,1 \mathrm{~ns}$ & 19,1 & & & $30,6^{* *}$ \\
\hline DMS & & 11,9 & 115 & 7,6 & -- & $32,5 * *$ & & & 0,9 \\
\hline C.V.(\%) & & 2,1 & 2,25 & 3,1 & 7,0 & 1,6 & & & 9,5 \\
\hline
\end{tabular}

(1) DAA= dias após a aplicação

${ }^{(3)}$ As médias na mesma coluna seguidas pela mesma letra, não diferem estatisticamente entre si, pelo teste de Tukey ao nível de 5\% de probabilidade. 
Tabela 4- Controle em percentagem das espécies encontradas na avaliação da seletividade e eficácia de misturas formuladas de bentazon acifluorfen em soja. Prudente de Morais, MG 1996/97.

\begin{tabular}{|c|c|c|c|c|c|c|c|c|c|c|}
\hline \multirow[t]{2}{*}{ Tratamentos } & \multirow{2}{*}{$\begin{array}{l}\text { Doses } \\
\text { i. } \text { a. }(\mathrm{g} / \mathrm{ha})\end{array}$} & \multicolumn{3}{|c|}{ Amaranthus hybridus ${ }^{(10}$} & \multicolumn{3}{|c|}{ Galinsoga parviflora $^{(I)}$} & \multicolumn{3}{|c|}{ Solanum americanum ${ }^{(I)}$} \\
\hline & & 15 DAA & $21 \mathrm{DAA}$ & 35 DAA & 15 DAA & 21 DAA & 35 DAA & 15 DAA & $21 \mathrm{DAA}$ & 35 DAA \\
\hline Volt & {$[480+204]$} & $97,2 c^{(2)}$ & $97,2 b$ & $96,5 \mathrm{c}$ & $96,2 \mathrm{~d}$ & $95,8 \mathrm{~cd}$ & $95,2 \mathrm{~d}$ & $96,5 \mathrm{~d}$ & $96,2 \mathrm{c}$ & $95,8 \mathrm{~d}$ \\
\hline Volt+Assist & {$[480+204]+0,25 \% \mathrm{v} / \mathrm{v}$} & $98,8 \mathrm{~b}$ & $98,8 \mathrm{~b}$ & $98,5 \mathrm{bc}$ & $97,5 \mathrm{bc}$ & $97,2 \mathrm{bc}$ & $97,0 \mathrm{bc}$ & $97,0 \mathrm{~cd}$ & $96,8 \mathrm{c}$ & $96,2 \mathrm{~cd}$ \\
\hline Volt & {$[600+255]$} & $98,5 \mathrm{bc}$ & $97,8 \mathrm{~b}$ & $97,5 \mathrm{bc}$ & $97,8 \mathrm{~b}$ & $97,5 \mathrm{bc}$ & $97,2 \mathrm{bc}$ & $98,0 \mathrm{bc}$ & $98,5 b$ & $98,5 b$ \\
\hline Volt+Assist & {$[600+255]+0,25 \% \mathrm{v} / \mathrm{v}$} & $98,5 \mathrm{bc}$ & $98,8 \mathrm{~b}$ & $99,0 \mathrm{~b}$ & $98,0 \mathrm{~b}$ & $97,8 \mathrm{~b}$ & $97,5 \mathrm{bc}$ & $98,8 \mathrm{~b}$ & $98,8 \mathrm{~b}$ & $98,5 b$ \\
\hline Gunner & {$[480+168]$} & $98,0 \mathrm{bc}$ & $97,8 \mathrm{~b}$ & $97,2 \mathrm{bc}$ & $97,2 \mathrm{cb}$ & $96,5 \mathrm{bcd}$ & $96,0 \mathrm{~cd}$ & $98,2 \mathrm{bc}$ & $97,5 \mathrm{bc}$ & $97,8 \mathrm{~b}$ \\
\hline Gunner+Assist & {$[480+168]+0,25 \% \mathrm{v} / \mathrm{v}$} & $99,0 \mathrm{~b}$ & $98,8 \mathrm{~b}$ & $98,5 \mathrm{bc}$ & $98,0 \mathrm{~b}$ & $97,8 \mathrm{~b}$ & $97,8 \mathrm{~b}$ & $98,8 \mathrm{~b}$ & $98,8 \mathrm{~b}$ & $98,5 b$ \\
\hline Gunner & {$[600+210]$} & $98,8 \mathrm{~b}$ & $98,8 \mathrm{~b}$ & $98,5 \mathrm{bc}$ & $97,8 \mathrm{~b}$ & $97,8 \mathrm{bcd}$ & $97,5 \mathrm{bc}$ & $98,8 \mathrm{~b}$ & $98,8 \mathrm{~b}$ & $98,5 b$ \\
\hline Gunner+Assist & {$[600+210]+0,25 \% \mathrm{v} / \mathrm{v}$} & $99,0 \mathrm{~b}$ & $98,8 \mathrm{~b}$ & $98,5 \mathrm{bc}$ & $98,0 \mathrm{~b}$ & $97,8 \mathrm{~b}$ & $97,5 \mathrm{bc}$ & $98,8 \mathrm{~b}$ & $98,8 \mathrm{~b}$ & $98,5 b$ \\
\hline Doble & {$[600+160]$} & $98,2 \mathrm{bc}$ & $98,2 b$ & $98,2 \mathrm{bc}$ & $96,5 \mathrm{~cd}$ & $96,2 b c$ & $96,0 \mathrm{~cd}$ & $98,8 \mathrm{bc}$ & $98,5 \mathrm{~b}$ & $98,2 b$ \\
\hline $\begin{array}{l}\text { Testemunha } \\
\text { capinada }\end{array}$ & ---- & $100,0 \mathrm{a}$ & $100,0 \mathrm{a}$ & $100,0 \mathrm{a}$ & $100,0 \mathrm{a}$ & $100,0 \mathrm{a}$ & $100,0 \mathrm{a}$ & $100,0 \mathrm{a}$ & $100,0 \mathrm{a}$ & $100,0 \mathrm{a}$ \\
\hline $\begin{array}{l}\text { Testemunha sem } \\
\text { capina }\end{array}$ & ---- & $0,0 \mathrm{~d}$ & $0,0 \mathrm{c}$ & $0,0 \mathrm{~d}$ & $0,0 \mathrm{e}$ & $0,0 \mathrm{e}$ & $0,0 \mathrm{e}$ & $0,0 \mathrm{e}$ & $0,0 \mathrm{~d}$ & $0,0 \mathrm{e}$ \\
\hline $\mathrm{N}^{0}$ indivíduos $/ \mathrm{m}^{2}$ & & 13,5 & 14,0 & 15,0 & 12,0 & 13,0 & 12,8 & 11,3 & 13,0 & 13,8 \\
\hline Valor de F & & $2123,2 * *$ & $1481,0 * *$ & $641,0 * *$ & $3624,2 * *$ & $1374,2 * *$ & $2360,4 * *$ & $1730,1 * *$ & $1452,3 * *$ & $1126,2 * *$ \\
\hline C.V.(\%) & & 1,4 & 7,8 & 2,6 & 1,1 & 1,8 & 1,4 & 1,6 & 1,8 & 2,0 \\
\hline
\end{tabular}

DAA $=$ dias após a aplicação

${ }^{(1)}$ Dados transformados em Arco seno $\sqrt{x / 100}$ para análise da variância

(2) As médias na mesma coluna seguidas pela mesma letra, não diferem estatisticamente entre si, pelo teste de Tukey ao nível de 5\% de probabilidade. 